\title{
INNOVATIONS IN PRIMARY CARE \\ Targeted Advanced Home Care for Patients With Moderate or Severe COVID-19
}

\author{
Koki Kato, $M D, M P H^{1,2}$ \\ Takafumi Nakagawa, $M D^{2,3}$ \\ 'Madoka Family Clinic, Ogori city, Fukuoka, Japan \\ ${ }^{2}$ Hokkaido Centre for Family Medicine, Sapporo city, Hokkaido, Japan \\ ${ }^{3}$ Sakaemachi Family Clinic, Sapporo city, Hokkaido, Japan \\ Ann Fam Med 2022;20:91. https://doi.org/10.1370/afm.2767.
}

\section{THE INNOVATION}

In Sapporo, Japan, coronavirus cases soared in May 2021, limiting the hospital's ability to admit moderate or severe cases quickly. In response to this situation, we organized a rapid response team to triage and treat, if necessary, home patients with coronavirus disease 2019 (COVID-19). Primary care physicians and home care nurses provided home visits and implemented home oxygen therapy and dexamethasone administration' for severe COVID-19 cases.

\section{WHO AND WHERE?}

This innovation was focused on home patients with moderate or severe COVID-19 in Sapporo city, Hokkaido, Japan. Sapporo Public Health Office designed the project supported by Hokkaido Disaster Medical Assistance Team (DMAT), clinics providing home visits, visiting nurse service agencies, and companies providing rental oxygen concentrators.

\section{HOW}

The public health officers conducted telephone triage for positive coronavirus cases. They obtained relevant information on risk factors such as age $>60$ years, smoking, diabetes, chronic kidney disease, cardiac disease, chronic lung disease, cancer, cerebrovascular disease, ${ }^{2}$ and risky symptoms such as shortness of breath and prolonged fever. Then, they sent information on patients with suspected severe COVID-19 to Sapporo Medical Association and DMAT, which had complete information on the availability of the inpatient beds for COVID-19 in Sapporo.

Conflicts of interest: authors report none.

\section{Corresponding author}

Koki Kato

Madoka Family Clinic

1-40, Asumi

Ogori-shi, Fukuoka 838-0109, Japan

koki.kato@hcfm.jp
If no beds were available, the DMAT contacted clinics to provide emergency home visits for those patients. Our practice, Sakaemachi Family Clinic, was one such clinic.

In response to a request from DMAT, we contacted each patient with suspected severe COVID-19 and provided home visits wearing personal protective equipment. At the time, we checked their oxygen saturation and symptoms and classified them into moderate, severe, or critical. ${ }^{1}$ For critical patients, we requested DMAT manage immediate hospitalization somehow. In other cases, we offered each patient a pulse oximeter ${ }^{3}$ and follow-up calls twice a day to monitor worsening signs and symptoms. We provided symptomatic treatment and home oxygen therapy with dexamethasone administration ${ }^{1}$ according to disease severity. Home care nurses responded to patients with fluid replacement therapy needs. Companies providing rental oxygen concentrators delivered the equipment to patients' homes.

We provided home visits for 17 patients during the period. Among those, 8 patients required immediate hospitalization; 9 patients received home oxygen therapy and dexamethasone administration; 3 patients needed delayed hospitalization after those therapies; 6 patients weaned from oxygen therapy and safely recovered at home.

\section{LEARNING}

The rapid response team for home patients with moderate or severe COVID-19 demonstrated satisfying results: timely home oxygen therapy and dexamethasone administration saved patients' lives. Hospitals for COVID-19 patients were tight worldwide due to the widespread delta variant. We hope that our experience will help worldwide primary care teams in the future in situations where they have to treat moderate and severe COVID-19 patients at home.

Read or post commentaries in response to this article.

Key words: home care services; telephone triage; COVID-19; oxygen

Submitted August 12, 2021; accepted September 1, 2021.

\section{References}

1. Horby P, Lim WS, Emberson JR, et al; RECOVERY Collaborative Group. Dexamethasone in hospitalized patients with Covid-19. N Engl J Med. 2021;384(8):693-704. 10.1056/NEJMoa2021436

2. World Health Organization. Clinical management of COVID-19: interim guidance. Published May 27, 2020. Accessed Aug 8, 2021. https://apps.who.int/ iris/handle/10665/332196

3. Shah S, Majmudar K, Stein A, et al. Novel use of home pulse oximetry monitoring in COVID-19 patients discharged from the emergency department identifies need for hospitalization. Acad Emerg Med. 2020;27(8):681-692. 10.1111/acem.14053 